\title{
Injury in the Developing World-From Prevention to Care
}

\author{
Karachi, Pakistan, 26-28 August 2008
}

The International Journal of Emergency Medicine publishes abstracts from various international meetings as a means to highlight ongoing international emergency medicine research.

The following abstracts are from the meeting "Injury in the Developing World-From Prevention to Care" held in Karachi, Pakistan on 26-28 August 2008. Injuries are a major public health problem in Pakistan and across the globe. Burden of disease analysis has shown injuries to be one of the leading causes of loss of healthy life. Injuries rank 11th in terms of mortality and second in terms of disability in Pakistan. The abstracts are organized into four main topic areas: (1) traffic accidents and injuries (2) domestic violence (3) public health and disaster medicine, and (4) general medicine.

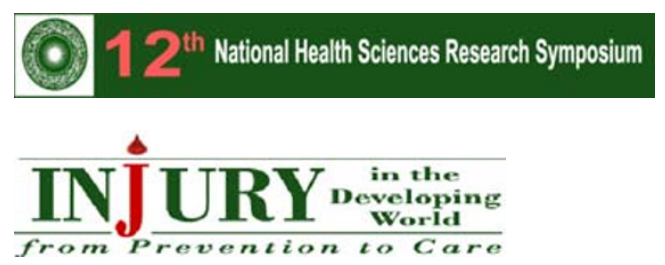

\section{TRAFFIC ACCIDENTS AND INJURIES}

Retirement transition: modification of driving behaviours and attitudes towards road safety

Junaid Ahmad Bhatti, Louis-Rachid Salmi

Institut de Santé Publique d'épidemiologie et de develloppement, France

Background In France, $60 \%$ of work-related injuries are due to road traffic collisions (RTCs). Analyzing behavioural and attitudinal changes associated with retirement can improve our understanding of occupational determinants of these RTCs. The objective is to assess the association between retirement transition and change in driving behaviours and attitudes toward road safety in the GAZEL cohort. Materials and methods GAZEL cohort, constituted in 1989, is composed of 20,000 employees of French utility companies. Two complementary analyses were performed using responses to questionnaires in 2001 and 2004: (1) The association between retirement in the 2001-2004 and change in risky driving behaviours and attitudes in the same period; (2) the association between time since retirement and risky driving behaviours and attitudes reported in 2001 . Results Twenty four percent of 10,612 participants retired between 2001 and 2004. Sixty three percent had retired before 2001. The results showed that those who retired between 2001 and 2004 were more likely to have changed towards less sleepy driving (OR=1.97 95\%CI [1.49-2.61]) and less cell phone use while driving $(\mathrm{OR}=1.69$ 95\% CI [1.07-2.38]) as compared to not retired participants (adjusted on socio-demographic and driving variables). In the second analysis, the proportion of risky driving behaviours decreased among retirees as a function of retirement distance, but changes occurred over longer time periods for speeding than for sleepy driving and cell phone use.

Conclusion Retirement is associated to safer driving behaviours. This implies a better evaluation of occupational determinants of RTCs.

\section{Factors associated with helmet use in Karachi}

Saad Shafqat, Imran Khan, Abdullah Khan

Aga Khan University, Pakistan

Objective Wearing a helmet is the single most effective measure for preventing head injuries in motorcycle users. We undertook this study to estimate compliance and determine reasons for non-compliance with helmet use among motorcyclists in our society.

Materials and methods This was a cross-sectional survey of motorcyclists in three large public-access parking spaces 
across Karachi, Pakistan's largest city. Questions covered personal demographics, frequency of helmet use, reasons for use or non-use, and knowledge of local helmet laws. Analysis was based on frequencies and group comparisons. Results Of the 300 (100\% male) subjects, 169 (56\%) reported using helmets regularly. Users listed injury prevention $(78 \%)$ as the major reason for compliance, while non-users listed physical discomfort (44\%), and limited vision $(25 \%)$ as the leading reasons for noncompliance. Helmet users were significantly more likely to believe that helmets are protective $(p=0.002)$, and that passengers should also wear helmets $(p<0.001)$. Several other variables (such as mean age, educational status, marital status, and knowledge of helmet laws) did not differ between users and non-users.

Conclusions Helmets are underused by motorcyclists in our society. This study underscores the need for improved helmet design, intense public education, and rigorous law enforcement in raising compliance with helmet use and minimising the risk of preventable trauma.

\section{Unintentional childhood injuries in semi-urban and rural communities of Sind and Baluchistan}

Seema Lasi, Ghazala Rafique

Aga Khan University School of Nursing, Pakistan

Background Injuries are a major cause of death and disability among young children. Every year, 750,000 children die from injuries. Another 400 million are seriously hurt. Many injuries lead to permanent disability and brain damage, which has lifelong consequences. Most of the injuries happen in or near home and almost all of them can be effectively prevented. The current study aims to determine the nature and extent of childhood injuries in two semi-urban and rural communities.

Materials and methods The findings of this study are based on a cohort of 2,201 children, 0-6 years old, in semiurban and rural areas of Sindh and Balochistan. Information was sought retrospectively from the primary caregiver (mother in most cases) about the occurrence of serious injury in or outside the house during the time since birth.

Results Cumulative prevalence of serious injuries, hospitalization and fatalities for children 0 to 6 years of age are reported here. The overall cumulative prevalence of serious injuries among children 0-6 years old was $9.7 \%$ with semiurban to be highest among the two areas with $16.4 \%$ prevalence. The male and female ratio for injuries was insignificant for both the areas. The most common type of injuries among children were fall from height (38\%), fall from stairs $(29 \%)$, scalds/burns $(12 \%)$ and road accidents
(05\%). Rate of Hospitalization was $03 \%$ among these children. Six of the primary caregivers also reported fatalities due to drowning (1), electric shock (1), road traffic accidents (2) and choking (2) among their offspring. Conclusion The burden of unintentional injuries is quite significant among these two areas. Fall from height or stairs came out to be the most frequent cause of childhood injuries. Majority of these injuries occurred in the house, which clearly shows the prevailing household hazards and inadequate measures of child proofing among these households. The recommendations are to educate families about the consequences of these childhood injuries and preventive measures to avoid them in future.

\section{A pilot-study of injuries among schoolchildren: the present situation}

Syed Muhammed Mubeen*, Abbas Subhani, Syed Aley Hasan Zaidi, Sadia Zia, Sumaira Afzal, Fatima Hussain, Munazza Naveed, Sana Majeed, Munazza Jawed

Hamdard College of Medicine and Dentistry, Hamdard University*, Pakistan

Objective The study explores the prevalence and type of injuries occurring in schools of Karachi. Surveillance and preparedness by the school staff is also highlighted.

Materials and methods A cross-sectional participatory cum observational study is carried out in both private and public sector schools using a self-administered questionnaire. Observations made by the students while collecting the data were also included. Forty-seven private and public sector schools of Karachi participated in the pilot study. Data analysis was done on SPSS ver 12.0.

Results Out of the 47 schools surveyed, 25 were private while 22 were in public (government) sector. Mean number of students for each class was found to be 33.0 (CI 95\% $25.4,40.7)$. Approximate number of injuries/accidents that occurred in 1 month was of 2.5 students (CI 95\% 1.6, 3.2). The two most commonly occurring injuries among students in both private and public school include a fall during playing and fighting/confrontation. Whereas the third common reason for injuries in public school was punishment by teachers, compared to injuries caused by fixtures in private schools. All school provided surveillance of students during their break timings; however, nearly $28 \%$ of both private and public schools denied the need of any surveillance for students. Record keeping of past injuries is almost non-existent.

Conclusion The common reasons of accidents/injuries among schoolchildren are mostly preventable. There is a need for proper teachers training for basic first aid skills. To 
keep a liaison with the nearest emergency centre along with proper record keeping is required.

\section{Trauma triage of the road traffic crash victims,} to the trauma centers of Karachi, Pakistan

\section{Sabeena Jalal Khan, Junaid A. Razzak}

\section{Aga Khan University, Pakistan}

Background Having an underdeveloped emergency medical system (EMS), there is no organized referral system for patients in Karachi, Pakistan which results in increased morbidity and mortality. Precious time can be saved if the patients were triaged to the closest trauma center.

Objective This study aims at determining the trend of trauma triage from a $1.5-\mathrm{km}$ radius range around the three major trauma centers of Karachi.

Materials and methods Data about road traffic crashes was collected from the medico legal records for the year 2003 because all injuries are to be reported to the medicolegal office by law. It was plotted on a vector map based on a satellite image using geographic information systems. An area of $1.5 \mathrm{~km}$ radius was selected so that the crash victims do not do not fall into overlapping areas.

Results The total road traffic crashes which occurred in Karachi were 3,650 victims, 1,435 (39.3\%) were rushed to Abbasi Shaheed Hospital (ASH), $883(24.2 \%)$ to Civil Hospital Karachi (CHK) and 1,332 (36.5\%) to Jinnah Postgraduate Medical Center (JPMC). Initial analysis shows that in an area of $1.5-\mathrm{km}$ radius around $\mathrm{ASH}$, there were 168 road traffic injuries (RTIs), while around CHK and JPMC there were 157 and 72 RTIs respectively. The range of the age for all the victims was $2-83$ years. The overall pre dominant population was male. In the area around $\mathrm{ASH}$, the mean age was (age $\pm \mathrm{SD}$ ) $33 \pm 16$ years, $81 \%$ victims were males. Out of 157 injuries around CHK the mean age was $34 \pm 17$ years, $89 \%$ were males. Around JPMC the mean age was $33 \pm 14$ years, $89 \%$ were males. From around ASH, 167 (99.4\%) were rushed to ASH, 1 $(0.6 \%)$ was taken to JPMC, who expired. Out of 157 RTI around CHK, 147 (94\%) were rushed to CHK and $10(6 \%)$ to JPMC. From the vicinity around JPMC, 66 (92\%) were taken to JPMC while $6(8 \%)$ were rushed to CHK.

Conclusion Even though there is no organized referral system here in Karachi, yet most of the injuries were being triaged to the nearest Tertiary care hospital.

\section{Violations of traffic rules at different posted speed limits in Malaysia}

Kulanthayan Subramaniam A. Kamarudin, T. H. Law

University Putra Malaysia, Malaysia
Abstract In Malaysia, there were 3.9 deaths per 10,000 registered vehicles in 2007. Police statistical records identify $15 \%$ of the road crashes to have occurred due to the element of speeding on the part of the vehicle driver. Therefore a study on the effects of different posted speed limits and violations of traffic rules was carried out on a major highway in Malaysia. Three stations were selected to represent threeposted speed limits 80,90 and $110 \mathrm{kmh}$. Both directions of the highway were included for speed observation. A total of 100 vehicles each from seven types of vehicles (motorcycle, car, taxi, light van and utility, medium lorry, heavy lorry and bus) were observed on each direction for their spot speed data during off peak hours, between 10 A.M. to 2 P.M., every day for 1 month .Data was analysed using SPSS by descriptive measures and chi-square test. Results from the descriptive test showed that the 85th percentile speed of car, taxi and buses was over the speed limits. The violations of speed traffic rules were higher among this group of vehicle drivers compared to motorcycle riders and drivers of light van and utility, medium and heavy lorry. More speed violations were also recorded among vehicles driving towards Central Business District (Kuala Lumpur City) area as compared to those outbound from Kuala Lumpur. Lastly, greater speed violations were recorded at $90 \mathrm{kmh}$ posted speed limit as compared to 80 and $110 \mathrm{kmh}$ posted limits. Therefore, road safety intervention programs should target these three identified factors.

Road traffic injuries and associated road-factors on Yaoundé-Douala road-section, Cameroon

Junaid Ahmed Bhatti, SobngwiI-Tambekou, L.-Rachid Salmi, Emmanuel Lagarde

Institut de Santé Publique d'épidemiologie et de develloppement, France

Introduction It is estimated that the African region has the highest road traffic injury (RTI) mortality rate. However, uncertainties regarding the actual size of the problem and associated epidemiological factors often jeopardize appropriate resource allocation.

Objectives To assess the incidence of RTIs on YaoundéDouala highway, Cameroon and investigate road-environmental factors associated with road traffic crash (RTC) sites. Materials and methods We obtained all available RTC reports $(N=935)$ from 13 police-stations along the roadsection $(243 \mathrm{~km})$. In addition, a case-control study assessing road-environment was carried out, where the 'case' $(N=$ 554) was a site involved in an RTC and the 'control' $(N=$ 544) was randomly selected from 1 to $10 \mathrm{~km}$ away from the RTC site towards Yaoundé. 
Results Over the 2004-2007 period, 374 persons died due to RTIs; the estimated number of deaths per 100 million kilometers travelled was 73 , a rate 35 times higher than that in Europe. The most severe crashes were those involving vulnerable road-users (97 deaths) and vehicles travelling in opposite directions (136 deaths). The main causes of fatalcrashes were hazardous overtaking (23\%), excessive speed $(20 \%)$ and mechanical failures $(28 \%)$, two-thirds being tire problems. The case-control site study showed that the following road factors were associated with the risk of RTCs; urbanization [adjusted odds ratio $(\mathrm{aOR})=1.37$ ], hills $(\mathrm{aOR}=0.66)$, poor road surface $(\mathrm{aOR}=1.40)$, obstacle $(<4 \mathrm{~m})$ from the road edge $(\mathrm{aOR}=2.20)$, and intersections $(\mathrm{aOR}=2.46)$.

Conclusion The burden of RTIs on highways in Cameroon calls for urgent interventions. Traffic calming on urbanizedsections, vehicle condition checks, and improvement of road-surface appear to be suitable interventions.

\section{A three-source capture-recapture study on traffic injuries in Kerman, Iran}

Seyed Abbas Motevalian, Kourosh Holakoie Naieni, Mahmood Mahmoodi, Reza Majdzadeh

School of Public Health, Iran University of Medical Sciences, Iran

Introduction In Iran, according to the National Death Registry, traffic injuries were the second major cause of death and first major cause of Years of Life Lost in 2001. The objective of the study was to estimate the number of deaths and non-fatal injuries applying capture-recapture method.

Materials and methods The data sources used for this capture-recapture study were: police, legal medicine organization and hospital. All of the deaths and non-fatal injuries due to traffic injuries that occurred within the Kerman district in 2001 were derived from the three data sources. Matching cases between the lists were based on three characteristics: first name, family name and date of accident. Statistical analysis included both two-source estimates and three-source analysis using Loglinear models. Results The total number of identified traffic deaths was 471; the best fitted loglinear model estimated the number of deaths as 596 (CI 95\%: 543-686); so the cause-specific mortality rate of traffic injuries was estimated as 92 (CI 95\%: 84-107) per 100,000 population. The sensitivity of police, legal medicine and Shahid Bahonar hospital and all three data sources was $16 \%, 58 \%, 48 \%$ and $79 \%$ respectively.

The best estimate for the number of non-fatal injuries was 10,682 (CI95\%: 5,192-13,843) which showed an incidence rate of 1.7 (CI95\%: 1.4-2.1) per 100 personyears. Sensitivity of police, legal medicine, hospital and all three data sources for reporting non-fatal traffic injuries was $7 \%, 7 \%, 63 \%$, and $70 \%$ respectively.

Conclusion The findings showed that applying capture recapture method will improve estimates of all deaths or nonfatal injuries.

\section{DOMESTIC VIOLENCE}

Prevalence of domestic violence before and during pregnancy: a prospective study from Hyderabad

Rozina Karmaliani, Farhana Madhani, Harris Hillary

Aga Khan University School of Nursing, Pakistan

Background Community-based descriptive studies on women's victimization of domestic violence among pregnant women from developing countries are scarce especially in Pakistan.

Objective To determine the prevalence and types of domestic violence affecting women before and during pregnancy living in Hyderabad, Pakistan.

Materials and methods To identify the prevalence of violence and its types prior and during pregnancy 1,368 women who were in their 20th to 26th weeks of pregnancy were interviewed using screening tool of 24 questions.

Results The results of this study show that women in Pakistan experience all types of violence at a high rate, with $47 \%$ of the women reporting some form of abuse 6 months prior to pregnancy and $38 \%$ reporting some form of abuse during pregnancy. Thirty-one percent of the respondents experienced only verbal abuse 6 months prior to the pregnancy and $16 \%$ of them experienced physical and sexual abuse. During pregnancy, $24 \%$ of the respondent experienced verbal abuse while $14 \%$ of them had physical and sexual abuse. The most common perpetrator was the husband followed by the motherin-law. In the majority of cases, women responded to the abuse by remaining quiet or verbally fighting back.

Conclusion The results of this study support the notion that domestic violence is prevalent among pregnant women in Pakistan. Widespread acceptance of patriarchal beliefs and the low status of women in Pakistan may explain these rates and the responses of women to violence. Preventative strategies should include education about violence and screening during health care encounters.

Domestic violence and its correlates among married women visiting family practice clinics in Karachi, Pakistan

Khuwaja AK

Aga Khan University, Pakistan

Background Domestic violence (DV) against women is a major preventable public health issue. It imparts hazardous effects on the physical, mental and social well-being of 
women who experience it, and children who witnesses it. The objective of this study was to assess the proportion of DV and its correlates in married women seeking services at family practice clinics in Karachi.

Materials and methods A cross-sectional survey was conducted on married women attending family practice clinics at four different sites in Karachi, affiliated with a private sector teaching hospital during the months of July and August 2005. A pre-tested, structured questionnaire was implemented to collect information from 400 married women, aged between 16 and 60 years, who agreed to participate in the study. Out of the total, 379 questionnaires were found to be complete for analysis. Multivariate logistic regression with $95 \%$ CI was used to identify the correlates of DV.

Results In all, 135 (35\%) women reported being ever violated by their husbands. Multivariate analysis disclosed significant associations between experiences of violence and women's illiteracy $(\mathrm{AOR}=5.9 ; 95 \% \mathrm{CI}: 1.4-25.1)$, husband's illiteracy ( $\mathrm{AOR}=3.9 ; 95 \% \mathrm{CI}: 1.2-12.5)$, husband being smoker (AOR $=3.4 ; 95 \% \mathrm{CI}: 1.2-9.4)$ and being substance user $(\mathrm{AOR}=3.4 ; 95 \% \mathrm{CI}$ : $1.8-6.6)$.

Conclusion Prevention of DV against women demands high priority along-with other preventable health issues. This study has revealed a significantly high burden of DV in married women in Karachi and has brought forth factors that can be used as foci for intervention strategies that are urgently required to prevent the devastating consequences of DV on women's health.

\section{Effect of domestic violence on pregnancy outcome}

Nabila Zareen, Nasreen Majid

Hamdard University Hospital, Pakistan

Objectives To identify the risk factors associated with domestic violence and to determine the effect of domestic violence on pregnancy, in terms of maternal and neonatal outcome.

\section{Design Cohort study}

Place and duration of study The study was conducted in Sir Syed Trust Hospital, from 1st March 2007 till 28th Feb 2008.

Materials and methods All antenatal patients were screened for domestic violence, using a self made questionnaire and Abuse Assessment Screen. All pregnant patients who had a language barrier, or those who declined to interview, were excluded from the study. All pregnant patients with a positive response according to the Abuse Assessment Screen were included in group A, while every fourth patient with negative response was taken as control. SPSS 10 was used for statistical analysis. Logistic regression analysis was performed to determine the effect of domestic violence on delivery outcome. Relative risk was also calculated. Students $T$ test was applied to compare means of numeric response variables between the two groups. Statistical significance was taken at $p<0.05$.

Results The risk factors for domestic violence included addiction in $39 \%$, aggressive nature of husband in $25.6 \%$ and unemployment of husband in $7.31 \%$ cases. Of the women, $52.43 \%$ had been victims to more than one form of violence, including physical, verbal and emotional abuse. Antenatal complications were not observed in a statistically significant manner in either group. Depression was identified as the main symptom in group A $(25.60 \%)$, though it was observed in only $3.65 \%$ in group B.

Conclusion Although various risk factors for domestic violence were observed in group $\mathrm{A}$, the difference between the two groups with regard to maternal complications and neonatal outcome (except depression) did not reach statistical significance.

Main outcome measures Types of violence, miscarriages, induced abortion, stillbirth, early neonatal death, low apgar score, preterm labour, Antepartum hemorrhage, depression. Keywords Domestic violence, Intimate partner violence, Maternal abuse, Abuse Assessment Screen.

\section{Domestic violence: a factor affecting women's quality of life}

Salma Amin Rattani

Aga Khan University, School of Nursing, Pakistan

Introduction Domestic violence is misuse of power in which an individual can be physically, sexually, emotionally and psychologically abused. Domestic violence is one of the factors that affects women's quality of life, as identified by the participants of the study entitled "A tool to assess the quality of life of working and non-working women in Karachi, Pakistan" (2006).

Objectives To explore the factors that affect women's quality of life.

Materials and methods A descriptive exploratory research design was employed. Using critical case sampling design, data was collected from three communities differentiated by their socio-economic standing i.e. low, middle and upper strata. Upon approval of Aga Khan University Ethics Review Committee and consent from the individual participants, 35 women were interviewed by using an interview guide. Descriptive analysis of data was done.

Results Women described domestic violence as one of the factors affecting their quality of life. Financial issue was voiced out to be one of the leading causes for domestic violence among women. Describing their experiences, women shared their ill feelings for being battered and traumatized.

Conclusions Unfortunately in Pakistan, due to social setup and societal acceptance, domestic violence is taken to be 
normal and personal issue of a family. Therefore, most of the time, it is not reported and no action is taken to reduce its incidence. It is important that domestic violence should be considered as a serious issue and strategies should be implemented to reduce its incidence.

\section{PUBLIC HEALTH AND DISASTER MEDICINE}

Decreasing vulnerability, increasing capacity: communitybased disaster management system as a public good

Atif Ikram Butt*, Bulgan Murun (Mongolia), Christina Kwauk (USA), Derek Lanter (USA), EiEi Khin (Myanmar), Kenichie Konaya (Japan), Thuan Le (Vietnam), Zhenwei Show (China)

United Nations Population Fund*, Pakistan

In a past few years, there has been an increase in the intensity of natural disasters inflicting utter devastation on a massive scale involving huge loss of human lives and material destruction. The lessons learnt from these disasters brought a painful realisation to the international community that natural calamities do not limit themselves to traditional national boundaries and make no distinction between rich and poor countries. However, while developed countries are better able to cope with coordinating and distributing immediate relief, maintaining early and sustained recovery, providing long-term reconstruction of the affected areas and building disaster resilient communities, it is the developing world which faces the brunt of the human and material losses.

One of the most crucial aspects of managing disasters, especially in the case of developing world, is a communitybased prevention and mitigation strategies and preparedness to respond immediately to a crisis. The first $24-72 \mathrm{~h}$ are especially critical because it is during this period that a community's own self-reliance becomes the main source of response. As a result, increasing a local community's capacity and resources that would enable it to immediately and effectively respond to natural hazards is a critical public good.

The paper, apart from developing a deeper understanding of the concept of 'public good' in relation to community-based disaster management and outlining the causes of the increase in natural disasters, argues that the rational explanation of communities lackluster involvement in disaster management lies in the concept of public good. Furthermore, the traditional top-down approach to natural disaster management cycle could be better complemented with the contemporary bottom-up community-based approach through enhanced understanding the characteristics, risks and limitations of the concept of public good.

The proposed paper is one of the outcomes of a 6-month long group project conducted under the Asia-Pacific Leadership Program at the East-West Center Hawaii.
Aga Khan hospital system, Pakistan's earthquake relief project in NWFP and AJK and the integrated health systems approach to post disaster relief efforts

Syed Zulfiqar Ali

Aga Khan Health Service, Pakistan

Background The October 8, 2005 earthquake resulted in massive destruction in AJK and NWFP. AKHS, P signed an agreement on 15th Oct, 2005 with UNICEF Pakistan for improvement of community health status of earthquake affected people through delivery of a comprehensive health care program as a joint venture by UNICEF, AKHS, P, Ministry of Health, Provincial and District Department of Health. The purpose of the paper is to highlight the strategy adopted by AKHSP in establishing a comprehensive health care system.

Methodology The four selected sites for the execution of the project were CH Garhi Habibullah and RHC Shinkiyari in Mansehra District, NWFP and RHC Arja (Bagh) and BHU Langerpura (Muzaffarabad) in AJK. AKHSP started the operations of these facilities after recruitment of the trained staff, and procuring the necessary equipment.

Results The service package comprised of facility and community based services. The total number of out patient visits during the project period is 93,844 , inpatient admissions 1,643, child vaccination for measles 3,089, tetanus toxoid administration 2,150, antenatal visits 3,657, family planning visits 1,226 and deliveries 236 . After a successful completion of its commitments AKHS, P exited and handed over the management of the CHCFs in NWFP to the DoH and to NRSP in AJK in consultation with DoH. Conclusion AKHS, $\mathrm{P}$ played a very important role in complementing the efforts of Government of Pakistan, international donors, $\mathrm{UN}$ and other agencies in promoting relief to the people and supporting revitalization of the health system. It adopted an integrated Health system approach instead of operationalising the damaged Government Health Centers rather than a parallel structure.

Experiences of nurses in disaster preparedness: lessons learnt from October 8, 2005 earthquake in Kashmir, Pakistan

Salima Bano Rafiq Virani, Laila Sewani

School of Nursing, Aga Khan University, Pakistan

Background Disaster preparedness is not just the understanding of types and components of disaster. In October 2005, when Pakistan was hit by a major earthquake, healthcare professionals from various Pakistani institutions actively participated in providing care to more than 4 million people who were affected from this catastrophe. 
Handling disaster on such a massive scale was a challenge to all. The purpose of this study was to explore the experiences of nurses, in regard to emergency preparedness in terms of managing mass casualties.

Materials and methods An explorative descriptive qualitative study design was used by conducting semi structured interviews. Sampling was purposive from those ten nurses who provided nursing care in the affected area. Qualitative aspects of data analysis were done with frequency and percentage.

Results The themes that were identified are:

1. Resource management and working with limited resources

2. Disaster management preparedness

3. Application of triage

4. Anticipatory concerns of participants, e.g. academic concerns and security concerns as being females

5. Care of health professional

6. Emotional response of nurses during and post disaster management

7. Challenges faced while providing care

Conclusion It is concluded that although nurses are efficient in dealing with emergency cases in the controlled setting of a hospital, they had difficulty to manage mass casualties in disaster hit areas. As this is a small scale explorative study there is a need to conduct of study on a larger sample size. This study recommends that emergency preparedness and disaster management should be the part of nursing curriculum. There should be short courses and hands-on workshop for registered nurses on how to triage and manage disaster in pre-hospital setting and in hospital setting.

Attitudes and perceptions of people towards suicide bombing - a cross-sectional survey in Karachi, Pakistan

Murad Moosa Khan*, Syed Faraz Kazim, Zarmeneh Aly, Bhisham Pradeep Harchandani, Affan Bin Irfan, Syed Mohammad Javed, Haider Khan Bangash, Rana Khalil-urRehman, Haider Naqvi

Aga Khan University, Pakistan

Introduction In recent years, suicide bombing has become a major problem in Pakistan causing large numbers of indiscriminate deaths and injuries as well as psychological trauma. Perpetuation of the act and recruitment of individuals in suicide bombing is influenced by general public's attitudes towards it.

Objective To study the attitudes and perceptions of Pakistani population towards suicide bombings.

Materials and methods A cross-sectional survey using convenient sampling method was done in Karachi, Pakistan's largest city (pop. approx. 15 million).
Results Two hundred fifteen people were interviewed, $49.8 \%$ males and 51.2\% females, 210 Muslims (73.5\% Sunnis and $24.2 \%$ Shias). $47.9 \%$ of the respondents were deeply religious, $42.8 \%$ restricted to rituals only, $9.3 \%$ agnostic.

Of the study respondents, $84.7 \%$ denied support for suicide bombing; only $15.3 \%$ supported it. The respondent's sex, age, marital status, level of education, and socioeconomic status did not show any significant association with the respondent's support for suicide bombing. All the $15.3 \%$ respondents who supported suicide bombing belonged to Sunni sect and were deeply religious and supported the involvement of religion in politics. Also all those people who supported that the concept of political struggle in Islam allows suicide bombing also supported the phenomenon of suicide bombing. Despite the fact that $84.7 \%$ of the study respondents did not support suicide bombing and $81.4 \%$ also denied that Islam or any other religion supported suicide bombing, $37.2 \%$ considered suicide bombing a martyrdom activity. $54.9 \%$ considered suicide bombers having some underlying psychiatric illness; $83.3 \%$ felt that suicide bombing was a result of religious fundamentalism.

Conclusions The majority of the respondents interviewed did not support suicide bombing but more than a third considered it as a martyrdom activity. There is a need to conduct similar studies with larger sample size in other regions and cities of Pakistan. The findings can lead to a better understanding of this controversial phenomenon.

Evaluation of levels and patterns of spinal cord injuries in the earthquake victims of October 8, 2005 in Pakistan

Shirin Mirza*, Syeda Fizza Tauqir, Shahzad Gul, Shahzad Gul, Asif Zafar Malik

Rawalpindi Medical College*, Pakistan

Objective To determine the extent of spinal cord injuries among patients affected by the earth quake of October 8th 2005 in Pakistan, through a cross sectional study.

Design Cross-sectional study

Place and duration of study The study was conducted from 10th October to 10th December 2005 in surgical and neurosurgical units of Rawalpindi Medical College and allied hospitals (Holy Family Hospital, Rawalpindi General Hospital and District Headquarter Hospital) and Melody Relief and Rehabilitation Center, Islamabad.

Materials and methods Level and patterns of spinal cord injuries was evaluated according to ASIA (American spinal injuries association) scoring system in 194 patients. Stability of spine and number of patients operated for spinal cord injuries was also assessed.

Results One hundred and ninety-four patients comprised of $74 \%(n=144)$ females and $26 \%(n=50)$ males. Seventyeight percent $(n=151)$ of the patients were of 16-39 years 
of age. Sixty-two percent $(n=120)$ of patients had injury at lumbar level, 25\% $(n=48)$ at thoracic level, 9\% $(n=18)$ at thoracolumbar level, a few had injuries at cervical and sacral level.46.4\% $(n=90)$ patients had spinal cord injuries which were graded " $\mathrm{A}$ " according to ASIA scoring system.4.12\% $(n=8)$ graded as "B", 10.82\% $(n=21)$ graded as "C", 9.2\% $(n=18)$ graded as "D" and $13.91 \%(n=27)$ graded as "E". Sixty-nine percent $(n=134)$ patients' spine was stable and $31 \%(n=60)$ had unstable spine. Seventyseven percent $(n=150)$ patients were operated and in $23 \%$ $(n=44)$ patients, operation was not indicated.

Conclusion Majority of spinal cord injuries occurred at thoracolumbar level leading to paraplegia. Those with ASIA score "A" would be bedridden for life. Proper rehabilitation programs should be inculcated to enable them to live an independent life.

Keywords Spinal cord injuries, levels, ASIA scoring system

Laparotomies among earthquake victims at Combined Military Hospital, Abbottabad

\section{Waqar Ahmed Khan, Shafquat Ali Abbasi \\ Combined Military Hospital, Pakistan}

Background On 8th October 2005 an earthquake measuring 7.6 on Richter scale jolted Northern Pakistan. It was one of the deadliest natural disasters of the recent history. Death toll was 74,500, and about 105,000 people were injured. An estimated 3.3 million were left homeless. In earthquakes of such magnitude, large numbers of severely injured patients are brought to health care facilities, which are usually insufficient to meet the requirements. Commonest causes of mortality in earthquake disasters are crush injuries, shock, head injuries, and in later phase multi-organ failure and renal failure. Combined Military Hospital (CMH) Abbottabad is a 500 bedded facility, which lies near the earthquake affected areas.

Results A total of 2,417 causalities were received and treated in the hospital from 8th Oct. 2005 to 31st Dec. 2005. Out of these 1,286 were hospitalized, and a total of 44 deaths were recorded. Abdominal-pelvic injuries were present in 95 patients. Sixteen out of these underwent laparotomies, these were analyzed retrospectively. A total of 975 serial ultrasound were done. A total of 16 laparotamics were done ding earthquake, out of these ten were done in first 2 days. Five laparotomies were negatives, only having fracture pelvis, so a more conservative policy was followed with serial ultrasounds and regular evaluation one patient was neglected, she got perforation and remained admitted in civil for 3 days due to the earthquake rush laparotomy and scared look also done but she could not survive. One patient forward to have caecal perforation a rare injury, two patients had ruptured bladder as they were sleeping after eating. Small leaks were covered as every patient was catheterized.

Conclusion It is recommended that in mass disaster situation a more conservative policy is safe for laparotomies to save time and energy for other serious patients A

\section{Managing a mass casualty situation at Army Medical Corps Training Centre, Abbottabad}

Waqar Ahmed Khan, Shafquat Ali Abbasi

Combined Military Hospital, Pakistan

Background On 8th October 2005 an earthquake measuring 7.6 on Richter scale jolted Northern Pakistan. It was one of the deadliest natural disasters of the recent history. Death toll was 74,500, and about 105,000 people were injured. An estimated 3.3 million were left homeless. In earthquakes of such magnitude, large numbers of severely injured patients are brought to health care facilities, which are usually insufficient to meet the requirements. Commonest causes of mortality in earthquake disasters are crush injuries, shock, head injuries, and in later phase multi-organ failure and renal failure. A military hospitals are designed and equipped to deal with wars and massive disasters. On 8 th October number of seriously injured patients started pouring in Combined Military Hospital (CMH) Abbottabad, which is a 500-bedded hospital and is located at the main draining road of the disaster struck valleys of earthquake affected areas. Disaster management plan was immediately activated and command post was established for administration and co-ordination purposes. Only three operation theaters, three surgeons and two anesthetists were available at the time of disaster.

Materials and methods During first week 751 seriously injured were hospitalized and required urgent surgical management, radiological investigations and laboratory support; thereby, putting enormous workload on the hospital. Therefore, all stable patients were discharged and OPD's were closed; two triage areas, trauma center consisting of 25 beds, four extra OT's, and additional post-op ward, were established within first $24 \mathrm{~h}$. Surgical team had to work round the clock for the first three days, after which assistance started arriving from other parts of the country.

Results It was only possible to increase 100 beds in hospital. All other medical facilities were teeming with patients so the only option left was to make camp arrangement in tents which was very difficult in winter. Army Medical Corps centre is located in vicinity of $\mathrm{CMH}$, voluntarily vacated the barracks and provided 450 patient beds and also looked after 1,500 attendants who had no place to go. A dressing room and a make shift OT was made in training school.

Conclusion This was a most secure arrangement for families and children. It is recommended that detailed plan must be chalked out in disaster management plans. 
Administering a mass casualty situation at Combined Military Hospital, Abbottabad

\section{Amjad Fahim, Shafquat Ali Abbasi \\ Combined Military Hospital, Pakistan}

Background Military hospitals are designed and equipped to deal with wars and massive disasters. On 8th October 2005, an earthquake measuring 7.6 on Richter scale struck North West Frontier Province and adjacent Kashmir; consequently large number of seriously injured patients started pouring in Combined Military Hospital (CMH) Abbottabad, which is a 500-bedded hospital and is located at the main draining road of the disaster struck valleys of earthquake affected areas. Disaster management plan was immediately activated and command post was established for administration and co-ordination purposes.

Results Only three operation theaters, three surgeons and two anesthetists were available at the time of disaster. During first week 751 seriously injured were hospitalized and required urgent surgical management, radiological investigations and laboratory support; thereby, putting enormous workload on the hospital. Therefore, all stable patients were discharged and OPD's were closed; two triage areas, trauma center consisting of 25 beds, four extra OT's, and additional post-op ward, were established within first $24 \mathrm{~h}$. Surgical team had to work round the clock for the first 3 days, after which assistance started arriving from other parts of the country. Additional wards with total 450 beds capacity were created in three regimental centers situated in neighborhood of CMH. Reserve medical stores of the hospital were utilized to cope up with the situation. In 3 months a total of 2,406 earthquake victims were received in the hospital, out of these 1,270 were admitted and 44 of these died, 15 and 12 of these were during the first and second weeks respectively, and total 54 amputations were done.

Conclusion Disaster management needs thread bare planning, correct coordination at all levels and execution through a well orchestrated system. Immediate mobilization of skilled manpower, equipment and medicines, training of health care workers and making of SOP's for dealing with mass disaster are required to save precious lives.

Pattern of injuries sustained in victims of earthquake, brought to the Emergency Relief Hospital

Fahad Ali, Fahad Sami

Aga Khan University, Pakistan

Objective Seismic waves can strike at any place any time. The knowledge of resulting injuries is important to deal with the present and future attacks so that respective preparations could be made.
This study was conducted to find out the pattern of injuries and their relationship with age and gender, to highlight the medical needs in an earth-quake affected zone. Materials and methods Cross sectional study, conducted during 13th Oct 2005 to 23rd Oct 2005 at the Emergency Relief hospital, Doraha, Mansehra.

Total 310 patients were included in the study, selected from the hospital admissions register by simple random sampling. Demography, and details of injuries were noted by history and physical examination.

Results Twelve cases were omitted due to incomplete data. Among the remaining 298 cases, 54\% were females. Children in the first decade of life formed the largest age group of injured patients. Isolated bony injuries were present in $41 \%$ of patients, soft tissue injuries in $36 \%$ and mixed injuries in $23 \%$. The most common bony injury was lower limb fracture (52\%) while the most common softtissue injury was non-infected soft tissue wounds on the limbs (33\%). Among all patients with soft tissue injuries, infected wounds were present in $30 \%$ and out of these, $20 \%$ were present on the limbs and 10\% were present on rest of the body parts. Gangrenous wounds were present in $9 \%$ of patients with soft tissue injuries.

Conclusion The population injured in the earthquake showed a higher proportion of females, a high number of children in the first decade of life and a high incidence of lower limb bony injuries. In the light of these observations, the orthopedic and pediatric surgeons, gynecologists and relevant diagnostic and treatment facilities be made at the initial stages of relief activities.

Earthquake paraplegics: where do they stand 1.5 years later?

Jawad Chishtie, Saifullah Khan, Farooq Azam Rathore, M. Umer

Subh-e-Nau, Pakistan

About 750 people were identified with sustained spinal cord injuries (SCI) in the October 05 earthquake. Their management was complicated by the inadequate rehabilitation services. Those returning homes did not have access to proper follow-up services.

Objectives To perform a follow up of earthquake 2005 paraplegics and assess their rehabilitation needs at the community level.

Materials and methods Cross-sectional study in Muzaffarabad district. Patients initially registered voluntarily on telephone and later in the field. Sixty-seven spinal cord injury patients were enrolled. A five-member team was formed that collected data in Feb. 2007. Interviews were conducted and examinations carried out. Data was entered on semi-structured proformas and analyzed using SPSS. 
Results Of the 67 patients enrolled, 42 patients had suffered an SCI (24 male; 18 female). Thirty were earthquake affectees, while 12 sustained injury due to falls or road traffic accident. Predominantly patients $(69 \%)$ were in their second and third decades, and had complete injuryASIA A (50\%). All were paraplegics with thoraco-lumbar injury. Pressure ulcers were present or had developed in eight, most with grade 2-3 ulcers. Co-morbid conditions were identified in $7 \%$, while $33 \%$ had at least one complication.

Recommendations/conclusions Early and adequate SCI rehabilitation leads to better outcomes, reduced complications. There is a need to improve community-based rehabilitation for the earthquake 2005 paraplegics to ensure their transition into mainstream society.

\section{GENERAL MEDICINE}

\section{Damage control in the abdomen-a review}

Kulsoom Faizullah, Hasnain Zafar

Aga Khan University, Pakistan

Background Damage control is not a modern concept, but the application of this approach represents a new paradigm in surgery, borne out of need to care for patients sustaining multiple high energy injuries. Multivisceral trauma and exanguinating haemorrhage lead to hypothermia, coagulopathy and acidosis. Formal resections and anastomoses lead to further physiological insults. Damage control addresses these physiological concerns by stage control and repair of these injuries.

Materials and methods All adult patients $(n=14)$ who underwent damage control laparotomy for trauma from January 01, 2000 to Date were identified in the medical records maintained in the Health Information Management Department at The Aga Khan University Hospital. The charts were retrospectively reviewed and outcomes were followed.

Results All 14 patients $(n=14)$ were men out of which eight $(n=8)$ expired.

Amongst the patients that expired four patients did not survive to reach the reoperation stage due to the severity of injuries, two were transferred in after primary packing about $24 \mathrm{~h}$ later and the cause of death was multisystem organ failure and the remaining two died of exanguination. Six patients $(n=6)$ are alive and doing well, out of these two developed adhesive small bowel obstruction, one had nonunion of tibial fracture, one had gluteal abscess, one had incisional hernia, and one has no morbidity to date.

Conclusion Damage control in the abdomen carries a high mortality rate. However, significant numbers are required to reach a definite diagnosis
Outcome of decompressive craniectomy in traumatic brain injury

Kishore Kumar, Fazal Wahab, Ehsan Bari, Kishore Kumar Aga Khan University, Pakistan

Introduction Morbidity and mortality of patients with severe traumatic brain injury remains high due to raised intracranial pressure (ICP) not responding to medical management. Surgical decompression is recommended in such cases. Although few studies on decompressive craniectomy have been reported, but there are no clearly defined criteria for selection of cases or appropriate timing of surgical intervention.

Objectives Assess the outcome of decompressive craniectomy in traumatic brain injury.

Materials and methods Audit, retrospective medical record reviewed for ICD 9 CM Code.

Duration 2004-2006

Number of patients Forty-five. Follow up 6 months. Outcome was based on Glasgow outcome scale and Karnofsky's score at discharge and at 6 months.

Result Out of 45 patients 13 were expired, 20 had good result and remaining is vegetative.

Conclusion Decompressive craniectomy is a good modality to treat refractory raised ICP. Initial GCS is strong predictor of outcome. Initial results are encouraging and compatible with International literature.

Penetrating thoracic trauma: presentation and management in Tertiary Care Centre

Vaqas Faroque, Hasanat Sharif

Aga Khan University, Pakistan

Introduction Thoracic injuries account for $20-25 \%$ of deaths due to trauma. Penetrating thoracic trauma accounts for almost one third of total thoracic trauma.

The mechanism of injury may be categorized as low, medium, or high velocity. Penetrations from blast fragments or from fragmentation weapons can be particularly destructive because of their extremely high velocities. Weapons designed specifically for antipersonnel effects (e.g., mines, grenades) can generate fragments with initial velocities of $4,500 \mathrm{ft} / \mathrm{s}$, a far greater speed than even most rifle bullets. The tremendous energy imparted to tissue from fragments with such velocity causes extensive disruptive and thermal tissue damage.

Objective To determine modes of presentation, management and outcome in penetrating chest trauma presenting to the Aga Khan University Hospital

Materials and methods A retrospective study over a period of five years from 2002 to 2007. All cases with penetrating thoracic trauma presenting to Aga Khan 
University Hospital. All charts presenting with the diagnosis of thoracic trauma were reviewed. All cases related to penetrating thoracic traumas were extracted. Patients were analyzed in three categories according to their presentation, time of presentation, time interval between events and their presentation, response time $\mathrm{b} / \mathrm{w}$ arrival and first visit, time between response and operation theater if needed and their operation theater dynamics regarding time of operation, duration of intubation, blood products needed etc. We also reviewed post operative HDU stay, over all stay, and out come with regard to morbidity and mortality. All patients were followed after their discharge. Data was analyzed in SPSS 14 Outcomes Out comes of our five year retrospection are:

- Most of the patients presented in Aga Khan hospital during 5 years are of young age ranging between 20 40 years

- Most of them are males (93\%).

- Commonest mechanism of injury is by gunshot (75\%).

- Average time of presentation is within $2 \mathrm{~h}(46 \%)$.

- Most thoracic injuries are managed conservatively.

- Most have a GCS>7 at presentation (97\%)

- Most common penetrations cause haemothorax and pneumothorax.

- Tube thoracostomy is the most frequent intervention $(62 \%)$.

- Most made a full recovery.

Recommendations

- Awareness regarding the penetrating thoracic trauma needs to be increased.

- All patients should be resuscitated with ATLS protocols

- Health care providers need to be aware of the golden hour.

- There is a dire need for proper and complete documentation.

\section{LIFE THREATENING CARDIOVASCULAR INJURIES}

Ghina Shamsi, Saulat Fatimi, Khalid Samad

Aga Khan University, Pakistan

Background Thoracic injuries are present in up to $75 \%$ of cases presenting with trauma. Ten to fifteen percent of these patients present with major cardiovascular trauma requiring urgent operative intervention to change the outcome. We define major cardiac and vascular trauma as thoracic trauma which are rapidly fatal if they are not promptly recognized and intervened.

Materials and methods We perform a retrospective audit of all major cardiac and vascular trauma presentation to emergency room of a tertiary care urban center over 5 years and their subsequent outcomes.

Results From January 2001 to December 2006, total of 168 patients were treated with thoracic trauma, of these 13 were recognized to have major cardiac and vascular injuries. Eighty percent of patients were males and commonly seen in second to third decade of life. Penetrating injuries were found to be the cause in $69 \%$ of cases, and associated injuries were found in more than $50 \%$ of the patients, the most common associated injury were abdominal injuries. Nearly $60 \%$ patients presented with either compromised circulation or airway and thus the clinical condition and judgment were the tools used for deciding to intervene. Subclavian vessels were the most frequent vascular structure that was insulted, five cases. The average time for presentations varied from 15 min for a bi-ventricular injury to up to $4 \mathrm{~h}$ for common carotid artery injury. The average time taken for patients to be shifted to the operating table was around $33 \mathrm{~min}$.

Conclusion The choice of incision was dictated by the injured organ and strongly influenced the outcome. The mortality rate in our series was $15 \%$. Early diagnoses, prompt transfer to the operating room and correct choice of incision influences the out come

\section{Characteristics of patients presenting with drug over- dose to a tertiary care hospital in Karachi}

Junaid Patel*, Muhammad Shahid, Mehmood Riaz, Imran Ayaz, Waqaruddin Kashif, Muhammad Tariq, Shoaib Khan, Jawwad Samdani

*Aga Khan University, Pakistan

Background Deliberate self harm with drugs and accidental overdose are common medical emergencies adding to the burden of diseases to any health care system. The aim of this study was to see the characteristics of patients admitted with drug overdose at a tertiary care hospital in Karachi.

Materials and methods A retrospective chart review was conducted at the Aga Khan University Hospital from January 2002 to October 2006. 324 adult patients admitted with drug overdose were included in the study. Data was entered and analyzed on SPSS.

Results Majority of patients were young (mean age 36 years) and women (59\%). The population included $34 \%$ housewives and $20 \%$ students. A significant number of patients who committed deliberate self harm (DSH) were married $(p=0.001)$ and had history of psychiatric illness. Domestic and social issues were the most common reason for DSH $(p=0.003)$. A good number of patients diagnosed on discharge as suffering from depression were females $(p=$ 0.028). Benzodiazepines were the most frequently used drug in both DSH (41\%) and accidental overdose (18\%).

Conclusion Our study shows that married women in particular housewives are at the greatest risk of DSH especially those with underlying depression. Domestic and 
social issues were rated highest among these individuals. Benzodiazepines were the most commonly involved agents in both DSH and accidental overdose. Most of our patients refused inpatient psychiatric treatment leading us to believe that general awareness of psychiatric illnesses is imperative in our community. The number of accidental overdose cases is alarming for us to consider modifying our existing prescription system.

Deconstructing myths and misconception regarding post surgical wound care among the patients visiting surgical ward of a tertiary care hospital: an approach towards patient education

Salma Molwani*, Naureen Hadwani

Department of Surgery, Aga Khan University, Pakistan

Objective To educate patients visiting the surgical ward of AKU regarding post operative wound care and management. Background Wound management has always been a major concern in any surgical patients because, if not healed properly it has many physical, social, financial and emotional implications for the patients as well as for the care givers. Although this fact was realized long before but proper management was, never possible due to patients having different myths and misconceptions regarding wound care and healing and application of such myths has always made the process of wound management complicated and difficult.

Materials and methods A cross sectional survey will be conducted through a validated questionnaire to find out the Knowledge, attitudes and practices of patients coming to the surgical unit of Aga Khan University Hospital regarding wound management. After assessing the KAP, educational material will be developed emphasizing on the areas identified by the KAP survey.

Conclusion After compilation of data on wound management, $80 \%$ patients prefer boil and low calorie diet, they think that water will infect their wound, they avoid to climb stairs and participate in social activities, even they were mentally feel upset and helpless all the time.

Seventy percent patients feel to take antibiotics even if the surgery has resulted in a clean wound. one more important observation that people are using home remedies to manage for care of wound, e.g. ash of cow stool (goober), egg yolk, paste turmeric and salt, paste of hot ghee and honey and many more.

Significant misconception about and healing is prevalent. These misconceptions should be challenged by education to alter/affect practices by patients and families.

The overall aim of this study is to shower some light on the existing myths, misconceptions and resulting malpractices adapted by patients for wound care and management and to educate them with high-quality research based educational information in the form of info booklets and pamphlets on dispelling those myths for quick and proper healing and management of wounds

Five years prospective audit of burns patients admitted to the ICU of a new burns unit in Karachi

Hashmi Madiha

Patel Hospital, Pakistan

Objective There is no organised Burns prevention or awareness program in Pakistan and deposit 60 years of development the human suffering from burns goes on unabated.

The purpose of this study was

1. To document the type and severity of burn along with mortality rate in patients admitted with serious burns to the burns-ICU of a new burns unit Karachi

2. To identify the individual most prone to get burnt, so that the burn hazard awareness program can be specifically targeted towards them and made more cost effective.

Materials and methods Date of all patients admitted to the burns ICU over a period of 5 years was collected prospectively by attaching audit forms to the files on admission.

Result There were 166 new admissions per year, male to female ration being 1:4:1. Eighty-one patients were less than 35 years old.

Fire burns were most common, kitchen being the most frequent place of accidents

Mortality increased with the percent of TBSA burnt

Conclusion and recommendation Emphasis on simple safety measures in the kitchen can prevent burns accidents in both females ( $80 \%$ of admissions) and males (25\% of admission)

Awareness of industrial and electrical safety measure may reduce accidental burns in young male at work. Children should be kept away from hot/boiled water to prevent scalds which is the most common cause of burns in this age group.

\section{CT scan assessment of traumatic brain injury and} potential impact on patient outcome

Shaista Afzal, Imrana Masroor,

Aga Khan University, Pakistan

Objective The aims of this study are to assess the frequency of abnormal CT findings in patients with traumatic brain injury and to evaluate the potential impact of these findings on patient outcome.

Materials and methods CT scan of all patients referred from ER with suspected traumatic brain injury were evaluated for midline shift, effacement of basal cisterns, subarachnoid hemorrhage, intra and extra axial hemorrhage, brain edema, fracture, soft tissue swelling and sinus 
opacification. The patient outcome was also noted and was categorized as unfavorable if these injuries were associated with neurological deficit or death and favorable if the patient's recovery was uneventful. Association of different CT findings with patient's outcome was also assessed.

Result The study was conducted in department of radiology AKUH for a period of 6 months and 166 patients were included .Out of the $166 \mathrm{CT}$ scans $86(52 \%)$ were normal without any findings and $80(48 \%)$ were abnormal. Among the patients with abnormal CT scans 26 (32\%) had unfavorable outcome and $54(68 \%)$ had favorable outcome. The CT findings associated with unfavorable outcome in this study were extra and intra axial hemorrhage, midline shift and effacement of cisterns. Single or few (up to three) findings were noted in patients' favorable outcome and multiple CT findings were noted in those with unfavorable outcome.

Conclusion The frequency of abnormal CT findings in patients with traumatic brain injury in our study is $48 \%$. The presence of multiple findings and intra and extra axial hemorrhage, midline shift and effacement of cisterns on CT scans obtained from patients suffering from traumatic brain injury are associated with unfavorable outcome and hence more aggressive therapeutic strategies are needed to avoid such outcome

\section{Complete spinal cord injury and role of surgery in developing countries}

Muhammad Shahzad Shamim, Syed Ather Enam, Muneeza Afif, Syed Faizan Ali

Aga Khan University, Pakistan

Objective To compare operative and non-operative management of patients with complete spinal cord injury with regards to clinical outcomes and cost effectiveness.

Materials and methods We devised a retrospective audit of all patients with complete spinal cord injury admitted at the Aga Khan University Hospital, Karachi from January 2004 till January 2007. All patients with traumatic complete spinal cord injury presenting within 14 days of injury were included. Patients were divided in two groups, those who underwent stabilization procedures and those who were managed non-operatively. The two groups were compared using independent samples $t$ test. The primary endpoints were (1) time to rehabilitation, (2) length of hospital stay, (3) 30 day morbidity or mortality, (4) cost of treatment and (5) long-term outcome.

Results Twenty-five patients fulfilled the inclusion criteria. Of these, 13 underwent operation. The two groups were comparable in terms of demographics. On comparison of the endpoints, patients in the operative group took a longer time to mobilize ( $p$ value 0.02 ); had longer hospital stays ( $p$ value $=0.006$ ), which included longer length of stay in special care unit ( $p$ value $=0.002)$ and ICU ( $p$ value $=$ 0.075 ); and had more complications ( $p$ value $=0.033$ ). The mean cost of treatment was also significantly higher in the operative group (USD 6,700) as compared to the nonoperated group (USD 1,450; $p$ value $=0.001)$.

Conclusion Significant differences were observed between the two groups. We recommend that patients with complete SCI in countries with limitations such as our own should be best managed non-operatively with a provision of surgery only if they remain symptomatic. Larger studies are recommended to further validate our results.

\section{Childhood injuries to chin, the undiagnosed mandibular condylar fractures}

Zafar Ali Khan, Jehan Alam, Hina Abid, Riaz Ahmed Warraich

King Edward Medical University and Allied Hospitals, Pakistan

Introduction/Objective Childhood injuries to the chin are common and may result in fracture of the mandibular condyle. If ignored or mismanaged these may subsequently result in temporomandibular joint (TMJ) ankylosis. The objective of this study was to document TMJ ankylosis resulting from trauma to the chin during childhood.

Materials and methods All patients presenting with TMJ ankylosis at the Oral and Maxillofacial Surgery Department of Mayo Hospital Lahore from July 2007 to March 2008 were included in this study. Patients' age, sex, socioeconomic status, previous history of injury to the chin (scar mark under the chin), level of health care center initially contacted and initial treatment received at the time of injury were documented.

Results A total of 60 patients presented with TMJ ankylosis, 34 (56.7\%) male and 26 (43.3\%) female patients with an age range of 4 to 35 years (mean 12.5 years). Fiftyeight $(96.7 \%)$ patients had a scar mark under their chin. Forty $(66.7 \%)$ had a history of fall from roof or a tree, 18 $(30 \%)$ were involved in a road traffic accident, all before the age of 10 years.

Recommendations/conclusions Trauma to the chin is common in growing age and, if ignored or mismanaged, can result in TMJ ankylosis. Poor socioeconomic status and lack of trained oral and maxillofacial surgeons plays a major role in delayed treatment of ignored cases. There is a need to create awareness among medical practitioners and general public about the long term outcome of undiagnosed and improperly/inadequately managed mandibular condylar fracture and consequent TMJ ankylosis.

Keywords Temporomandibular joint, Ankylosis, Trauma, Mandibular condylar fracture, childhood 
Attitudes and perceptions of people toward suicide bombing in tribal areas of Pakistan-a questionnaire survey

Abdul Wahab Yousafzai, Mohammed Irfan, Abdul Waheed Khan, Arshad Iqbal

Aga Khan University, Pakistan

Introduction Suicide bombing (SB) is a new form of terrorism in Pakistan that started in November 2002. Following the military operations in 2004, there has been a progressive increase in SB in tribal areas, with more than 2,500 deaths in over 90 suicide attacks in Pakistan. Tribal areas have been portrayed as training bases and breeding ground for SB. This study aims to explore public attitudes and perceptions toward SB in tribal areas of Pakistan.

Materials and methods This was a cross-sectional study using an 18-item questionnaire developed after an extensive literature search, focus-group discussions and experts' opinion. Data was managed using SPSS version 15. Descriptive statistics were used to calculate frequencies and tabulate the information.

Results Out of 446 participants, 78\% were males. Mean age of the participants was 29 years. Majority (75\%) were Pashto speaking and 61\% were educated. Eighty-one of respondents felt that Islam should play a decisive role in the thought process of Muslims, 75\% preferred to live in an Islamic state but $52 \%$ felt that Pakistan is not an Islamic country.

An overwhelming majority (93\%) argued that 'Jihad' is a religious obligation for Muslims while $87 \%$ disagreed that suicide bombing was the result of Islamic fundamentalism and said that they do not support it. Ninety percent condemned suicide bombing. Seventy-three percent of the respondents viewed suicide bombing as an 'international conspiracy' against Pakistan. About the common man's attitude, $87 \%$ of the respondents said that people in Pakistan strongly oppose suicide bombing and $88.8 \%$ said that there is 'no justification' for suicide attacks in Pakistan. About characteristics of suicide bombers, opinions varied: $28 \%$ said suicide bombers were illiterate, $23 \%$ felt they came from poor background and $12 \%$ considered suicide bombers as religious fanatics.

Conclusions People in tribal areas of Pakistan, although strongly inclined toward religion and consider jihad as a religious obligation, do not consider suicide bombing a legitimate action. These views have important implications as extremist behaviors like SB cannot be sustained without public support. Prevention strategies agaisnt SB must be broad based and incorporate local public opinion towards SB.

Keywords Suicide bombing, Pakistan, Tribal areas, attitude

\section{Principles of imaging in trauma}

Vaqar Bari

Aga Khan University, Pakistan

Abstract: The role of various imaging modalities will be discussed with examples and images of various important conditions

- Learn about:

- Types of Imaging (X-ray) studies used for trauma patients

- Understand their uses

- Examples of organ and skeletal injury and typical findings

- Cervical spine

- Head

- Chest

- Abdomen

The importance of qualitative research in identifying strategies to reduce deaths due to drowning

Rasheda Khan, A. A. Hyder, S. E. Arifeen, Sabina Shahnaz

International Centre for Diarrhoeal Disease Research, Bangladesh (ICDDR,B), Bangladesh

Objective Behavioral change messages, developed through qualitative research, are part of the package of interventions for reducing injuries. Social science methods aid in understanding the explanatory models of injuries and designing feasible and acceptable strategies to reduce injuries and deaths. This study was carried out to understand the cultural context of drowning and identify strategies to reduce drowning deaths in Bangladesh.

Materials and methods The study was carried out in Matlab, Bangladesh. Methods included cognitive mapping, in-depth interviews and group discussion.

Results The application of three qualitative research methods allowed for the creation of an explanatory model for drowning. Explanation for drowning is often associated with evil spirits. Care providers perceive noon to be the most difficult time to supervise young children. Mothers are perceived to be the primary care providers. A variety of strategies used to protect children from drowning were also reported through these methods. For possible interventions, a number of preventative strategies like daycare centers, leashes, playpens, carrying the child on back, door barrier and swimming were explored. Findings suggest that people were most responsive to playpens and door barriers while least responsive to carrying the child on back.

Conclusion Cultural aspects and social frameworks need to be taken into account to ensure that strategies and messages 
devised for injury control are relevant, acceptable and appropriate. Injury specialists need to make use of qualitative insights to devise approaches that strike a balance between health ideology and social customs on one hand, and sound

\section{Pattern of injuries in high mountain villages of northern Pakistan: a community-based study}

Ghulam M. Hyder, Syed M. Shah, Rehman Alvi, Abid Hussain Aga Khan Health Services, Northern Areas, Gilgit, Pakistan

Objective The study was carried out to measure the burden of injury in order to provide data for use in developing strategies for injury prevention efforts.

Materials and methods We conducted a community-based household survey to determine non-communicable risk factors during 2007 in Punyal valley of Northern Areas, Pakistan. A representative sample of 1,000 households was selected to participate, using probability-proportional-tosize methods. A respondent was randomly selected from household members aged 18 years and over. Information on injuries occurring in the past 12 months preceding the interview was collected using a standard questionnaire.

Results The crude incidence of injuries was $6.4 \%$ in total, and it was higher in men (11.8\%) than in women (5.9\%). After adjustment of age, income and education men were more likely to be injured compared to women (adjusted odds ration 2.01, 95\% CI $0.99-4.45, p$ value $<0.05$ ). A significant proportion of injuries $(40.2 \%)$ were caused by farm related activities. More than half (52.4\%) were hospitalized for injury. Majority (44.4\%) of injuries were sustained due to fall from a tree or other elevation, followed by road traffic accident $(12.7 \%)$, operating a machine ( $8 \%$ ), slip on a wet or uneven surface $(8 \%)$, hit by an animal (6.3\%), burn (3.2\%) and others (17.4).

Conclusion Injury is a significant public health problem among residents of the rural North and seriously burdens health care systems and families. Development of injury prevention strategies need to focus on determinants of injuries.

\section{Pattern of obstetrical injuries at public sector university hospital of Sind, Pakistan}

Meharunnissa Khaskheli, Imdad A. Khushk

Liaquat University of Medical and Health Sciences, Jamshoro

Objective To find out the frequency, types and complications of obstetrical injuries during labour in our set up.

Place and duration This study was conducted at Department of Gynaecology and Obstetrics (Unit-IV) Liaquat University of Medical and Health Sciences, Jamshoro from June 2006 to May 2007.
Study design A descriptive study

Materials and methods All the women who delivered vaginally and by cesarean section and suffered from obstetrical injuries during study period were included in the study all those women who had normal vaginal delivery and cesarean section without any obstetrical injuries were excluded from the study. Case records of all these women were thoroughly analysed regarding their age, parity, gestational period, mode of onset of labour, handling by TBA, duration of labour, mode of labour, types of complication, their management and outcome.

Results Frequency of obstetrical injury with vaginal delivery was $8.76 \%$ and with abdominal delivery it was $5.59 \%$. Common types of injuries were perineal tear in 24 (36.36\%) cases, vaginal tear in $19(28.78 \%)$ cases, cervical tear in $13(19.69 \%)$ cases and bladder injury in $2(11.76 \%)$ cases. Complications included post partum haemorrhage in $24(36.36 \%)$ cases, shock in $6(9.09 \%)$ cases, recto vaginal fistula $1(1.51 \%)$, septicaemia in $1(5.88 \%)$ and death in 1 (5.88\%).

Conclusion The frequency of obstetrical injury is high during delivery at our set up. To avoid this, there is strong need for identification of risk factors, planned delivery, timely decision for any intervention so as to encourage women to consider delivery positively and adverse out come must be minimized.

\section{Practices regarding diagnosis and management of third and fourth degree perineal tears}

Raheela Mohsin Rizvi, Nasreen Chaudhury

Aga Khan University, Pakistan

Objective To review our practice of diagnosing and managing perineal tears.

Materials and methods Retrospective case series conducted by reviewing medical records of all women having singleton, alive, full term vaginal delivery at Aga Khan University Hospital, Karachi from November 1999 to October 2005.

Results The frequency of perineal tears in our department was $0.5 \%$ out of a total of 26,844 vaginal deliveries. A large majority of women (75.5\%) were nulliparous. Eighty six patients had forceps delivery (63.7\%) out of 135 patients. In only 28 patients $(20.7 \%)$, the diagnostic criterion for classification of third degree tears was used. For the repair of third degree tear end-to-end method was performed on $97(71.9 \%)$ patients. Twenty women had subsequent uncomplicated vaginal deliveries and none of these patients were subjected to endo-anal ultrasonography and/or anal manometry while the documented evidence regarding planning of future delivery was found in only four cases. 
Conclusion In order to find out the exact incidence of perineal tears the use of standard classification of perineal tears is strongly recommended. The use of episiotomy or forceps can be selective. Future mode of delivery should be carefully planned after complete evaluation.

Keywords perineal tears, diagnosis, management

Frequency and determinants of child abuse reported by their mothers in family practice clinics in Karachi, Pakistan

Niloufer Sultan Ali, Ali Khan Khuwaja

Aga Khan University, Pakistan

Objectives Globally, child abuse is one of the major public health as well as clinical problems. This study was conducted to evaluate the frequency of child abuse and to identify its determinants as reported by mothers in Karachi, Pakistan.

Materials and methods A cross-sectional study was conducted in Family Medicine clinics affiliated with a private sector, tertiary care teaching hospital in Karachi,
Pakistan. After giving consent to participate in the study, 379 mothers were interviewed by trained data collectors. A pre-tested and structured questionnaire was used comprising different sections including socio-demographic, mother's personal and marital life, and violence against herself and the children. Chi-square test was used to identify the various determinants associated with child abuse.

Results Over one quarter $25.5 \%$ of children were abused physically and $17.9 \%$ children were abused verbally. In all, $32.5 \%$ of children had experienced at least any form of abuse. The two most significant factors identified with child abuse were abused mother (abused mother: $50.4 \%$, nonabused mother: $21.7 \%$; $p<0.001)$ and having female child (female child: $62.5 \%$, male child: $45.5 \% ; p<0.001$ ). However, mother's education $(p=0.045)$, father's education $(p=0.027)$ and father's working $(p=0.004)$ status (office job and business versus manual work and labor) were found to be protective determinants towards child abuse.

Conclusion This study revealed that one-third of the children were physically and/or verbally abused. Interventions are suggested to prevent this problem. More studies are required to explore this important health issue in more detail. 